\title{
IDENTIFYING HIGH-METALLICITY M GIANTS AT INTRAGROUP DISTANCES WITH SLOAN DIGITAL SKY SURVEY
}

\author{
Lauren E. Palladino ${ }^{1}$, Kelly Holley-Bockelmann ${ }^{1,2}$, Heather Morrison $^{3}$, Patrick R. Durrell $^{4}$, Robin Ciardullo ${ }^{5,6}$,

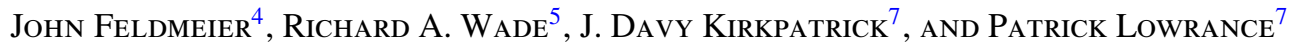 \\ ${ }^{1}$ Department of Physics and Astronomy, Vanderbilt University, Nashville, TN 37235, USA; lauren.e.palladino.1@ vanderbilt.edu, k.holley@ vanderbilt.edu \\ ${ }^{2}$ Department of Natural Sciences and Mathematics, Fisk University, Nashville, TN 37208, USA \\ ${ }^{3}$ Department of Astronomy, Case Western Reserve University, Cleveland, OH 44106, USA \\ ${ }^{4}$ Department of Physics and Astronomy, Youngstown State University, Youngstown, OH 44555, USA \\ ${ }^{5}$ Department of Astronomy and Astrophysics, The Pennsylvania State University, University Park, PA 16802, USA \\ ${ }^{6}$ Institute for Gravitation and the Cosmos, The Pennsylvania State University, University Park, PA 16802, USA \\ ${ }^{7}$ Infrared Processing and Analysis Center, MS 100-22, California Institute of Technology, Pasadena, CA 91125, USA \\ Received 2011 July 18; accepted 2012 February 8; published 2012 April 30
}

\begin{abstract}
Tidal stripping and three-body interactions with the central supermassive black hole may eject stars from the Milky Way. These stars would comprise a set of "intragroup" stars that trace the past history of interactions in our galactic neighborhood. Using the Sloan Digital Sky Survey DR7, we identify candidate solar-metallicity red giant intragroup stars using color cuts that are designed to exclude nearby M and L dwarfs. We present 677 intragroup candidates that are selected between $300 \mathrm{kpc}$ and $2 \mathrm{Mpc}$, and are either the reddest intragroup candidates (M7-M10) or are L dwarfs at larger distances than previously detected.
\end{abstract}

Key words: Galaxy: halo - Galaxy: stellar content - Local Group - stars: late-type

Online-only material: machine-readable and VO tables

\section{INTRODUCTION}

A significant fraction of the stellar component of a galaxy cluster is not confined to any galaxy. These stars between galaxies form luminous halos, called intracluster light (ICL), with very low surface brightness that can extend out to several hundred kiloparsecs around individual galaxies (e.g., Abadi et al. 2006; Krick \& Bernstein 2007). The brightest ICL is less than $1 \%$ of the brightness of the night sky (Mihos 2003; Feldmeier et al. 2004b), thus making a complete census of ICL very difficult to obtain. High resolution $N$-body simulations estimate that ICL could comprise $10 \%-70 \%$ of the total cluster luminosity (Mihos 2003; Murante et al. 2004).

It is commonly thought that intracluster stars are caused by one of three main channels: (1) stripping from galaxies as the cluster assembles either via high speed galaxy encounters, tidal shocking, or a rapidly changing galaxy cluster potential (Byrd \& Valtonen 1990; Merritt 1984), (2) long-lived, low level cluster perturbations in the form of "galaxy harassment" (Moore et al. 1996), or (3) tidal stripping within in-falling galaxy groups (Mihos 2004; Rudick et al. 2009). These processes will generate a stellar "debris field" that is highly inhomogeneous, with distinctly non-Gaussian velocities that reflect an unrelaxed intracluster population (Napolitano et al. 2003). Thus far, ICL has been identified via planetary nebulae (PNe; Feldmeier et al. 2004b; Aguerri et al. 2005), Red Giant Branch stars (Durrell et al. 2002; Williams et al. 2007), intracluster globular clusters (Lee et al. 2010; Peng et al. 2011), and ultra-deep surface photometry (Feldmeier et al. 2002, 2004a; Mihos et al. 2005).

The properties of ICL may provide insights into the accretion history and evolution of galaxy clusters (Mihos 2003, 2004; Napolitano et al. 2003; Feldmeier et al. 2004b; Conroy et al. 2007). Although there is some debate about the role that tidal stripping plays in ICL production, it is expected that ICL substructure is correlated with the dynamical state of the cluster (Murante et al. 2007; Mihos 2004). Since the vast majority of galaxies reside in poor groups, rather than in large clusters, it is of great interest to determine the fraction of unbound stars that reside in these environments.

In the Local Group, ICL has not been observed, though deep observations and star counts have revealed a "field of streams" (e.g., Belokurov et al. 2006). These streams have been detected out to $100 \mathrm{kpc}$ and are bound to the Milky Way (e.g., Yanny et al. 2000; Ibata et al. 2001). Similarly, faint streams have been detected in the outskirts of M31 (McConnachie et al. 2009; Ibata et al. 2007). Given that the Milky Way and M31 are not yet interacting and may not even be part of the same dark matter halo, it is more likely that Local Group ICL, if it exists, would be a product of a different process altogether.

One of the more recent suggestions for ICL production is via three-body interactions (Holley-Bockelmann et al. 2005). For example, stars can be thrown out from the galaxy through tidal disruption of a binary star system by a supermassive black hole (SMBH; Hills 1988; Yu \& Tremaine 2003); this is the most common explanation for "hypervelocity" stars such as SDSS J090745.0+0204507, with a galactic rest frame velocity of $\sim 700 \mathrm{~km} \mathrm{~s}^{-1}$ (Brown et al. 2005). During this process, energy and angular momentum are transferred from the black hole to one of the stars in the binary. The second star loses energy and becomes bound to the black hole while the first is ejected from the galaxy. This is expected to occur at a rate of $10^{-5}(\eta / 0.1) \mathrm{yr}^{-1}$, where $\eta$ is the stellar binary fraction (Magorrian \& Tremaine 1999).

Another three-body interaction that is likely to expel stars is a close encounter of a single star with a binary black hole (Yu \& Tremaine 2003). This is expected to occur at a rate of $10^{-4}(\eta / 0.1) \mathrm{yr}^{-1}$ (Magorrian \& Tremaine 1999). In this case, the star gains energy from the binary black hole and is flung out of the galaxy while the black hole orbit shrinks (e.g., Quinlan 1996; Sesana et al. 2006). 
To become gravitationally unbound, stars must exceed the escape velocity of the Galaxy, now estimated to be $500-600 \mathrm{~km} \mathrm{~s}^{-1}$ (e.g., Smith et al. 2007). Semi-analytic models predict that there may be approximately 100 hypervelocity stars within $8 \mathrm{kpc}$ of the galactic center if the binary stars have equal masses (Yu \& Tremaine 2003). However, intragroup stars (IGS) may not be solely comprised of hypervelocity stars; they may still be bound but on very large, highly eccentric orbits - this can increase the potential number of IGS. One way to get stars on such eccentric orbits is through three-body galaxy ejections of satellites like Leo I (Sales et al. 2007; Mateo et al. 2008).

As a first attempt to probe for a population of intragroup stars, we develop a technique to search for $\mathrm{M}$ giant stars in between the Local Group galaxies. In this paper we present our technique for identifying candidate IGS from the Sloan Digital Sky Survey (SDSS) by applying color, distance, and proper motion cuts. Section 2 describes our technique. We present our results in Section 3, and we discuss possible sources of contamination in Section 4. Section 5 concludes and discusses methods to confirm the candidates.

\section{METHODS}

During its eight years of operation, the SDSS (York et al. 2000) obtained deep, multi-colored images covering more than a quarter of the sky. The SDSS uses five optical bandpasses ( $u, g, r, i$, and $z$; Fukugita et al. 1996; Gunn et al. 1998; Hogg et al. 2001; Gunn et al. 2006) with magnitude limits 22.0, 22.2, $22.2,21.3$, and 20.5, respectively. The DR7 data set contains $12,000 \mathrm{deg}^{2}$ of images and a catalog of over 350 million objects with spectra of 460,000 stars.

As individual red giant stars in M31 have been observed down to the SDSS magnitude limits (e.g., Zucker et al. 2007), intragroup red giant stars will be observable. Indeed, at a distance of $300 \mathrm{kpc}$, all supergiants and roughly half of any giants would be detectable in the $r, i$, and $z$ bands.

We developed our technique using the synthetic SDSS and 2 Micron All Sky Survey (2MASS) photometry (Covey et al. 2007) of flux calibrated spectra of solar-metallicity main sequence, giant, and supergiant standard stars (Pickles 1998). With simulated SDSS and 2MASS colors (Schlegel et al. 1998), we obtained a stellar locus to search for giants or supergiants that are isolated in a "clean" region of color space. These color-color diagrams are shown in Figure 1. We choose solar-metallicity standards to probe for IGS generated by three-body interactions within the central regions of the Galaxy, as discussed in Section 1.

Unfortunately, most supergiants and giants lie along the mainsequence locus and would therefore be indistinguishable by SDSS colors. However, there is a small area in each of the color-color diagrams, shown with dashed boxes in Figure 1, where the rarest $\mathrm{M}$ giants (M7-M10) are isolated from dwarfs and supergiants of the same spectral types. Considering the distances we are probing and the very red colors of these spectral types, we restrict our color selection to the $r, i$, and $z$ bands as follows:

$$
\begin{aligned}
& 2.1<r-i<3.4, \\
& 1.3<i-z<2.2 .
\end{aligned}
$$

Again, we drop the $u-g$ and $g-r$ cuts because objects at these distances are likely too faint in these bands.
Since these objects are so red, they may be confused with other non-stellar objects. ${ }^{8}$ However, we find that even quasars with $z>4.6$ are too blue in $i-z$ to fall in our color space (Richards et al. 2002; Fan et al. 2001).

A more worrisome source of contamination comes from $\mathrm{L}$ and $\mathrm{T}$ dwarfs. To investigate this, we compared our color selection region to the colors of $\mathrm{L}$ and $\mathrm{T}$ dwarfs (Hawley et al. 2002) and find that they also are contained in the color region, albeit with large uncertainties, as shown in Figure 2. From current observational studies, we estimate that there may be $O(1000)$ early L dwarfs in the SDSS footprint within the magnitude limits of SDSS (Burgasser et al. 2010). Since we expect more dwarfs than giants at these faint magnitudes, it is likely that a greater number of dwarfs are scattered into the selection area through large errors than the number of giants scattered out. Objects in our selection box that are not IGS are, nevertheless, likely interesting astrophysical objects yielding more distant $\mathrm{L}$ dwarfs than currently known. We discuss ways to differentiate between IGS and contaminants in Section 5.

From the DR7 Star Table, we identified all stars that satisfy our color criteria and are positioned at $|b|>20$ to exclude potential disk contamination (including disk $\mathrm{L}$ and $\mathrm{T}$ dwarfs). To ensure that each candidate has a stellar point spread function, we confirmed that the object type flag in all five bandpasses were stellar. ${ }^{9}$ We then removed all objects that would be nearer than $300 \mathrm{kpc}$ and further than $2 \mathrm{Mpc}$ in the redder bandpasses, assuming an $\mathbf{M}$ giant average absolute magnitude in each bandpass $\left(M_{r}=0.8, M_{i}=-1.5, M_{z}=-3.5\right)$ yielding the following magnitude cuts: $23.2<r, 20.9<i, 18.9<z$ - since the $r$ band is effectively below the magnitude limits of SDSS, we searched for null detections in this band as well. We also eliminated objects that triggered any of the following flags: BADSKY, BLENDED, CHILD, COSMIC_RAY, EDGE, MAYBE_CR, MAYBE_EGHOST, MOVED, NODEBLEND, and PSF_FLUX_INTERP.

We cross-checked our candidates with the 2MASS $J-H$ color cut of Majewski et al. (2003) since dwarfs and giants have distinctive $J-H$ colors: $J-K_{s}>0.85, J-H<$ $0.561\left(J-K_{s}\right)+0.36, J-H>0.561\left(J-K_{s}\right)+0.22$ (top panel of Figure 3). Although, note that the Majewski et al. (2003) color cut selects sub-solar-metallicity M giants with $[\mathrm{Fe} / \mathrm{H}]=-0.4 \pm 1.1 \mathrm{dex}$ (Chou et al. 2007). This will make the giants selected by the Majewski et al. (2003) cut bluer than the solar-metallicity giants selected in our sample.

The middle panel of Figure 3 shows a clear separation of the synthetic spectral standard giants and dwarfs from Pickles (1998) at a $J-H$ color around 0.8 (Bessell \& Brett 1988). In general, $J, H$, and $K_{s}$ magnitudes for our IGS candidates (16.86, 15.78, and 15.56, respectively, for an M7III according to Covey et al. 2007) are too faint to appear in the 2MASS catalog. Since appearing in 2MASS would indicate that the source is too bright

\footnotetext{
8 Background red galaxies have colors roughly $0.35<r-i<0.45$ and $0.15<i-z<0.3$ at $z=0.1$ (Blanton et al. 2003). These colors will become redder with increasing redshift. For example, the brightest and reddest galaxies in SDSS, Luminous Red Galaxies (LRGs) at $z=0.5$, have $r-i=0.7$ and $i-z=0.4$ (Blanton et al. 2003). Since these colors do not approach our color selection region and since we have ensured stellar point spread functions for our candidates, we do not consider background galaxies as a significant source of contamination.

9 Although we restrict our color criteria to the $r, i$, and $z$ bands, in order to be conservative, we still require the objects to have stellar point spread functions in the $u$ and $g$ bands as well.
} 

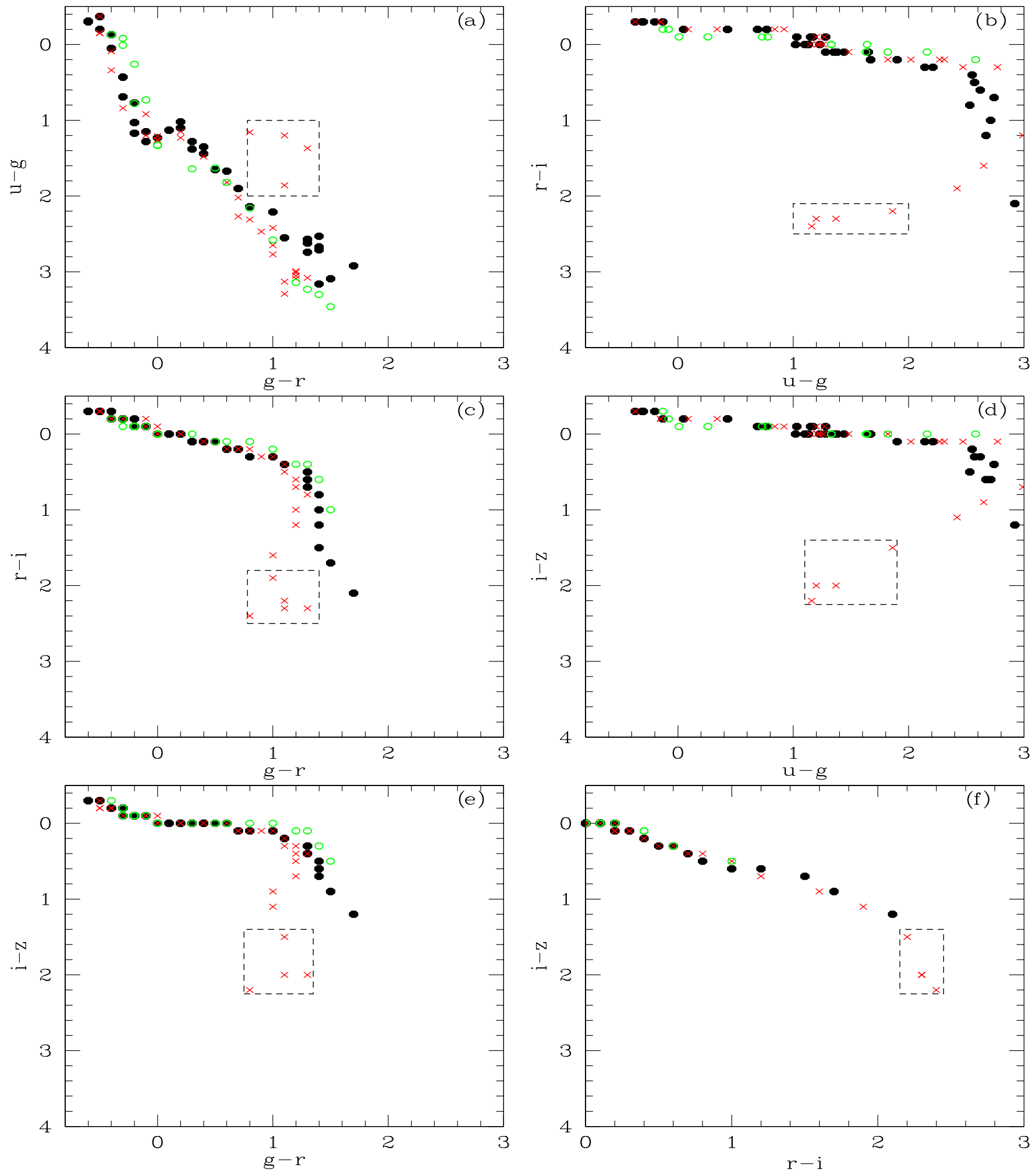

Figure 1. Color-color diagrams of the Covey et al. (2007) sample data. Main-sequence stars are marked with black dots, giants with red crosses, and supergiants with green circles. Note that the reddest giants (M7-M10), identified by the black dashed regions, are isolated from the stellar locus. This figure is a theoretical demonstration that very late $\mathrm{M}$ giants have the potential to be isolated in color space in the ugriz bands, and these dashed regions are meant only to highlight the spectral types of interest and do not represent our color selection criteria (given explicitly in Equations (1) and (2)). In particular, we drop the $u-g$ and $g-r$ cuts because objects at these distances are likely too faint in the $u$ and $g$ bands. The locus of sub-solar-metallicity giants is generally indistinguishable from the dwarf locus, and thus not plotted here.

or too close to be an IGS M giant we removed any candidate that did appear in the 2MASS catalog, and to verify that they are nearby dwarfs, we plotted their $J-H$ colors shown in the bottom panel of Figure 3.
The completeness limits of $J, H$, and $K$ filters in the UKIDSS (Lawrence et al. 2007) survey are appropriate for our targets, however our search through the publicly released database (DR4) did not result in any matches to our candidates since 

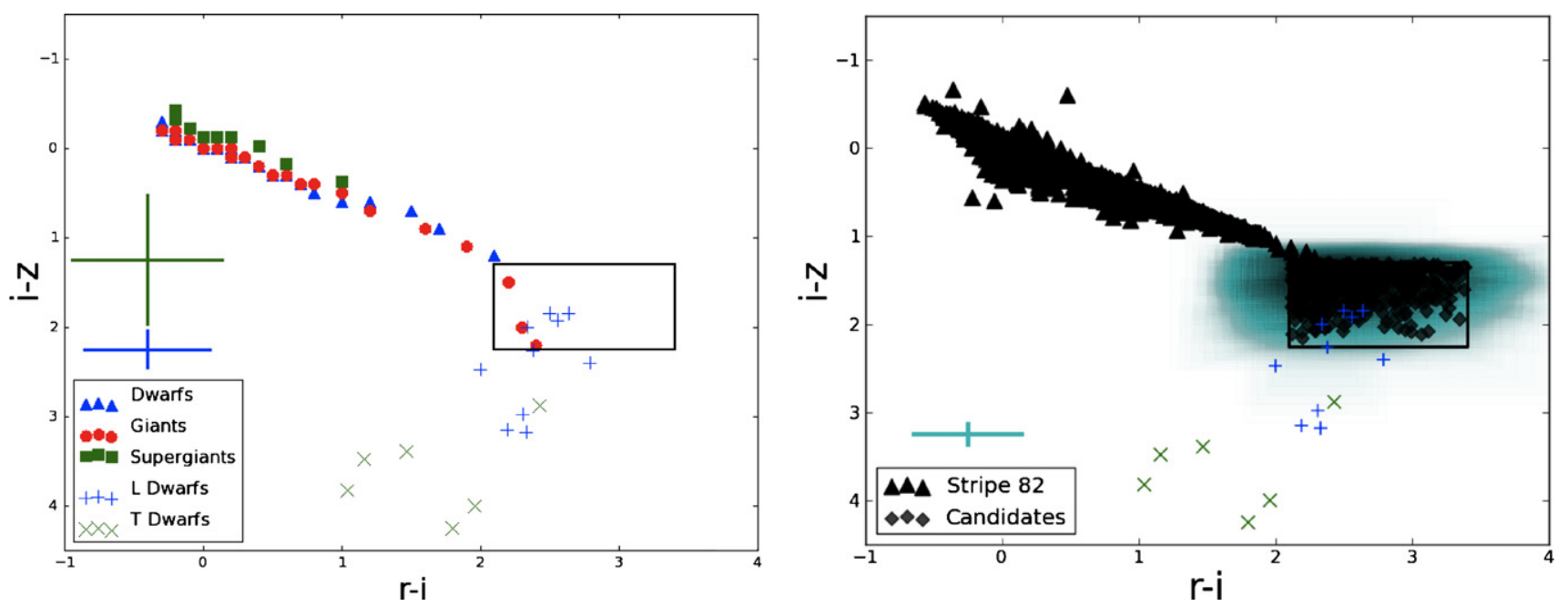

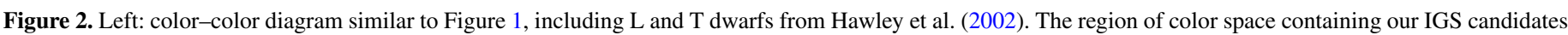

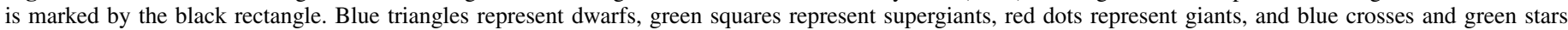

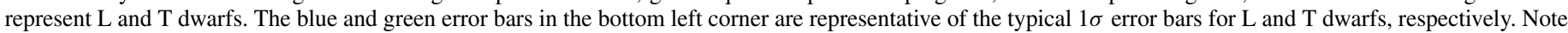

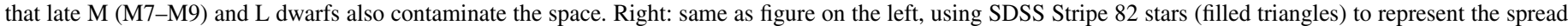

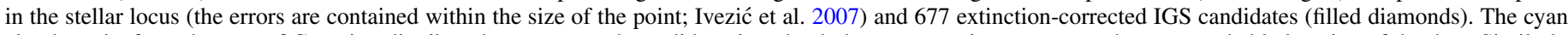

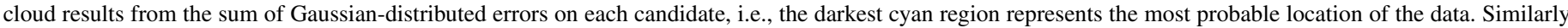
as in the left panel, the cyan error bar in the bottom left corner is representative of the typical $1 \sigma$ error bars for each candidate.

we expect our candidates lie in parts of the sky not yet covered by this release. Moreover, once the UKIDSS survey is complete it will only cover about half of the area in the SDSS footprint.

After removing known dwarfs in 2MASS, we crossreferenced the IGS candidates with the USNO-B catalog, an all-sky catalog that contains positions, proper motions, and magnitudes in various optical passbands. Here, we removed those candidates with discernible proper motions, ranging from 3 to 1412 mas year $^{-1}$, since the distances determined by the proper motions indicate that they are likely objects closer than $353 \mathrm{pc}$. This eliminated 782 candidates.

\subsection{Testing the Color Selection Region with Real Data}

Since our current technique for finding very late $\mathrm{M}$ giant IGS is based on idealized solar-metallicity spectra and synthetic colors, it is important to determine how robust these colors are for known M7III-M10III stars. Unfortunately, there are no confirmed very late $\mathrm{M}$ giant stars in the SDSS DR7 (or DR8) database with both spectra and colors. While it is true that these stars are rare, the real difficulty is that spectral classification of $\mathrm{M}$ giants is notoriously difficult, and the latest $\mathrm{M}$ giants can be spectral type variables, as well. Since the SDSS database did not explicitly include the latest $M$ giants, we decided to take a three-pronged approach in checking $\mathbf{M}$ giant colors.

First, we searched through Simbad for spectroscopically confirmed M7-M10 giants, finding 53, 28, 4, and 0, respectively; many of these were listed in the Catalogue of Stellar Spectral Classifications (Skiff 2010). For each object, we cross-checked the spectral type through all other publically available catalogs on VizieR to determine if the star was a known significant spectral variable, and if so, we discarded it. We then searched DR7 for any star within 2 arcsec of the target and obtained the photometry. Many of these objects were nearby and saturated, and therefore appeared as several non-stellar sources in the DR7 database - these were also discarded if the composite, corrected photometry was not available. Only one of the remaining stars returned a result in the Catalog Archive Server (CAS) and appears as the blue star in Figure 4, which lies within our color cut.

Second, we acquired spectra of late-type giants observed as part of a study to quantify the effects of gravity on the spectra of cool objects. These spectra were obtained with the Low Resolution Imaging Spectrometer (LRIS; Oke et al. 1995) on the $10 \mathrm{~m} \mathrm{~W}$. M. Keck Observatory as part of a campaign to construct a systematic surface gravity "grid" to further constrain spectral classifications of brown dwarfs (J. D. Kirkpatrick et al., in preparation). We estimated the spectral type and $\log (g)$ parameters by eye and separated the latest $M$ giant subset for analysis, totaling 13 spectra. Examples of these spectra are shown in Figure 5. Using the SDSS transmission curves, we calculated the colors in the Sloan bands, and as can be seen by the red stars in Figure 4, most of these stars do indeed lie in the predicted color space.

Finally, as a further check of our $\mathrm{M}$ giant colors we used the Bruzual-Persson-Gunn-Stryker (BPGS, hereafter; Laidler et al. 2005) stellar atlas of standard stars to obtain synthetic colors using the IRAF Synphot task calcphot $^{10}$; the green points in Figure 4 represent these standard stars - there were no available M9III-M10III standards in the atlas, which is expected because these objects are all spectrum variables.

Figure 4 shows that the color cut we defined from synthetic SDSS colors is consistent with the colors of known $M$ giants from all three techniques.

If the reader is interested in seeing the colors of the stars we used from the BPGS stellar atlas in the Johnson-Cousins filter set, a figure will be available online. ${ }^{11}$ The $U B V$ colors of these stars are consistent with the colors reported by Worthey \& Lee (2006), as well.

\section{RESULTS}

We found a small region of color space, shown in Figure 2, in which the reddest solar-metallicity $M$ giants are isolated from

\footnotetext{
10 The $i-z$ colors returned by calcphot for the M dwarfs are unreliable due to negative flux values in the BPGS stellar atlas.

11 http://astro.phy.vanderbilt.edu/ palladl2/
} 
Table 1

IGS Candidates Remaining After All Criteria Cuts

\begin{tabular}{|c|c|c|c|c|c|c|c|c|c|c|c|c|c|c|}
\hline Object ID & R.A. & Decl. & $u$ & $u$ err & $g$ & $g$ err & $r$ & $r$ err & $i$ & $i$ err & $z$ & $z$ err & $r-i$ & $i-z$ \\
\hline 758882136836343139 & 60.5013 & 80.8760 & 24.4 & 1.3 & 24.7 & 0.6 & 24.3 & 0.7 & 22.0 & 0.2 & 20.3 & 0.2 & 2.3 & 1.7 \\
\hline 758882137910740030 & 62.9212 & 82.5298 & 25.2 & 1.2 & 25.0 & 0.6 & 23.2 & 0.4 & 21.0 & 0.1 & 19.6 & 0.1 & 2.2 & 1.4 \\
\hline 758882626993718584 & 63.3170 & 81.6950 & 26.5 & 0.6 & 24.8 & 0.6 & 25.3 & 0.7 & 22.0 & 0.2 & 20.3 & 0.2 & 3.3 & 1.6 \\
\hline 758877527803168329 & 94.6323 & 63.7038 & 25.1 & 0.9 & 24.1 & 0.3 & 24.5 & 0.5 & 21.2 & 0.1 & 19.8 & 0.1 & 3.3 & 1.4 \\
\hline 758877527266231607 & 94.8017 & 63.2633 & 24.6 & 0.7 & 24.4 & 0.5 & 24.5 & 0.6 & 21.8 & 0.1 & 20.4 & 0.1 & 2.7 & 1.4 \\
\hline 758877527266493955 & 95.9369 & 63.5501 & 25.2 & 0.7 & 24.8 & 0.6 & 25.6 & 0.5 & 22.7 & 0.3 & 20.7 & 0.2 & 2.9 & 2.0 \\
\hline 758878272976455144 & 105.3459 & 66.8553 & 25.3 & 0.9 & 24.4 & 0.4 & 24.1 & 0.4 & 21.8 & 0.1 & 20.2 & 0.1 & 2.3 & 1.6 \\
\hline 758878271902778853 & 105.8427 & 66.0785 & 23.8 & 0.7 & 24.7 & 0.4 & 24.6 & 0.5 & 22.3 & 0.2 & 20.8 & 0.2 & 2.3 & 1.5 \\
\hline 758884768580109918 & 107.9936 & 38.3022 & 24.8 & 1.1 & 25.1 & 0.7 & 24.4 & 0.6 & 21.9 & 0.1 & 20.4 & 0.2 & 2.6 & 1.4 \\
\hline 587738067260998978 & 109.5721 & 39.4395 & 25.3 & 0.9 & 25.7 & 0.6 & 24.6 & 0.7 & 22.1 & 0.2 & 20.0 & 0.1 & 2.5 & 2.1 \\
\hline
\end{tabular}

(This table is available in its entirety in machine-readable and Virtual Observatory (VO) forms in the online journal. A portion is shown here for guidance regarding its form and content.)
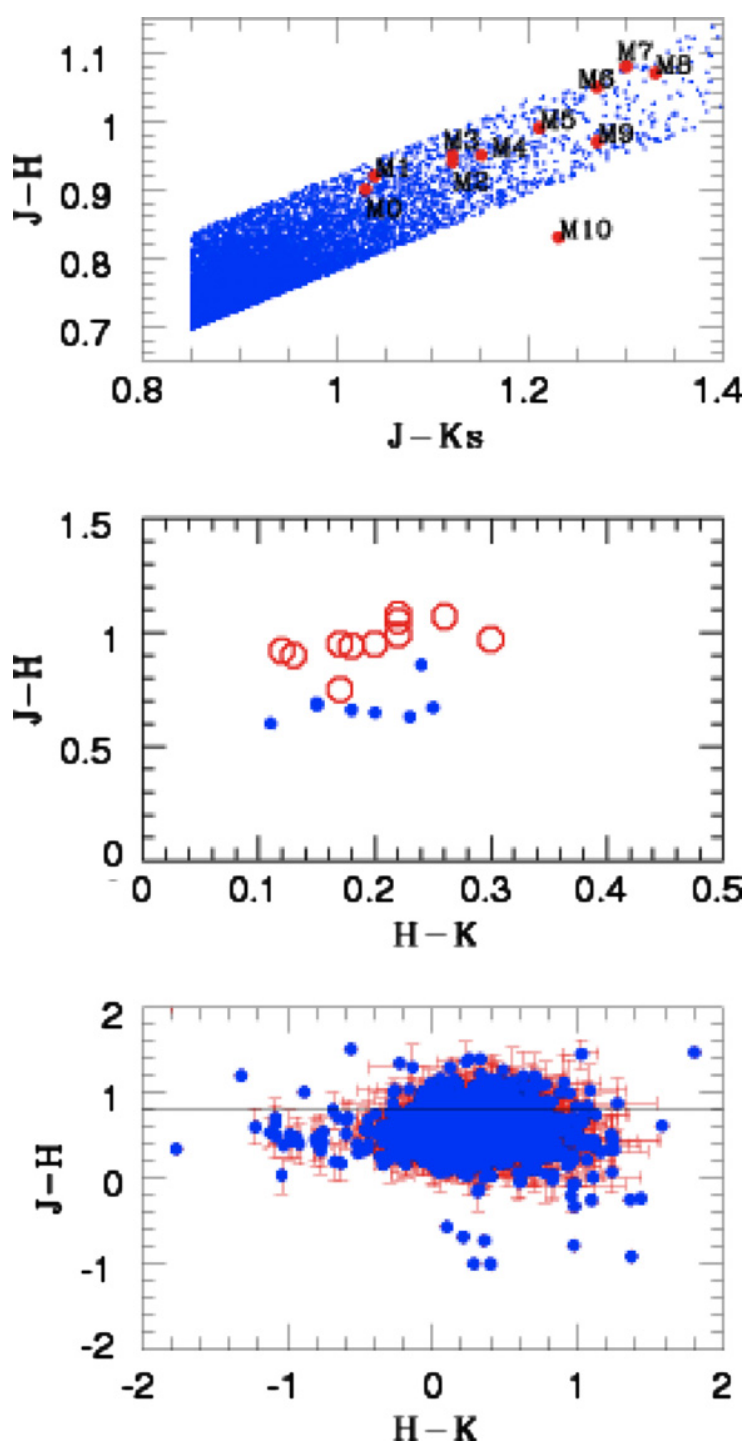

Figure 3. Top: blue dots are SDSS Stripe 82 stars (Ivezić et al. 2007) that satisfy the Majewski et al. (2003) M giant color cuts. Larger red dots are M giants from Covey et al. (2007). The point located outside the color cut corresponds to an M10 spectral type. Also note that the late M giants (M7-M10) are located in the part of this color region that is least populated, so they will be least likely to be identified by this cut. Middle: red open circles are M giants from Covey et al. (2007). Blue dots are M dwarfs from Covey et al. (2007). Bottom: IGS candidates with 2MASS $J H K$ colors. The solid line represents the $J-H=0.8$ separation between dwarfs and giants.

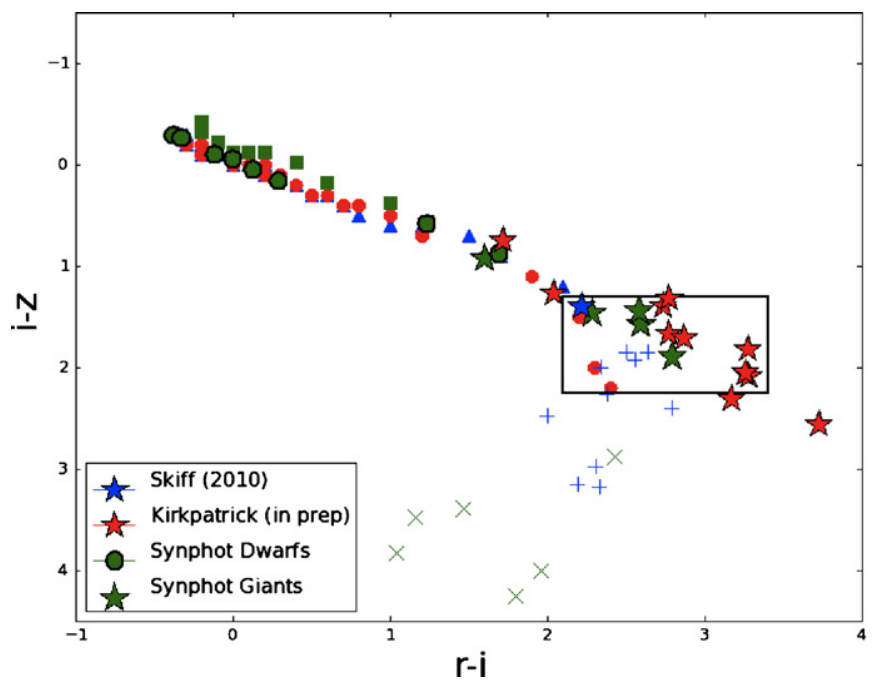

Figure 4. Same as the left panel of Figure 2. The green stars overplotted here represent M-giant standards, with spectral types between M6III and M8III, from the BPGS stellar atlas. The larger green dots are dwarfs with spectral types $\mathrm{O}$ through M, from the same atlas. The photometry for these stars was obtained by implementing IRAF synphot tasks. The blue star represents the $\mathrm{M}$ giant identified from the Catalogue of Stellar Spectral Classifications (Skiff 2010) with confirmed SDSS photometry. The red stars are the M giant contaminants in the J. D. Kirkpatrick et al. (in preparation) data for which we received spectra.

the rest of the stellar locus. This region hosts M7III-M10III stars, along with L dwarfs (Hawley et al. 2002).

Using our color selection criteria, we found 159,108 extinction-corrected objects in SDSS DR7. After applying the distance cut and checking the data flags, we narrowed the sample to 4181 objects. We then cross-correlated our sample with the 2MASS and USNO-B surveys, removing any stars with dwarf-like $J-H$ colors and any stars with non-zero proper motions. Our final sample contains 677 IGS candidates. Table 1 lists positions, asinh magnitudes, $r-i$, and $i-z$ colors for all 677 candidates. The right panel of Figure 2 shows the location of the final set of IGS candidates with errors in $r-i / i-z$ color space.

\section{DISCUSSION}

As discussed in Section 1, IGS could be formed from several different methods. Considering the Local Group's current level of interactions, this population may likely be comprised of highmetallicity hypervelocity stars (HVS) ejected through the threebody mechanism. However, not enough is known about HVS 


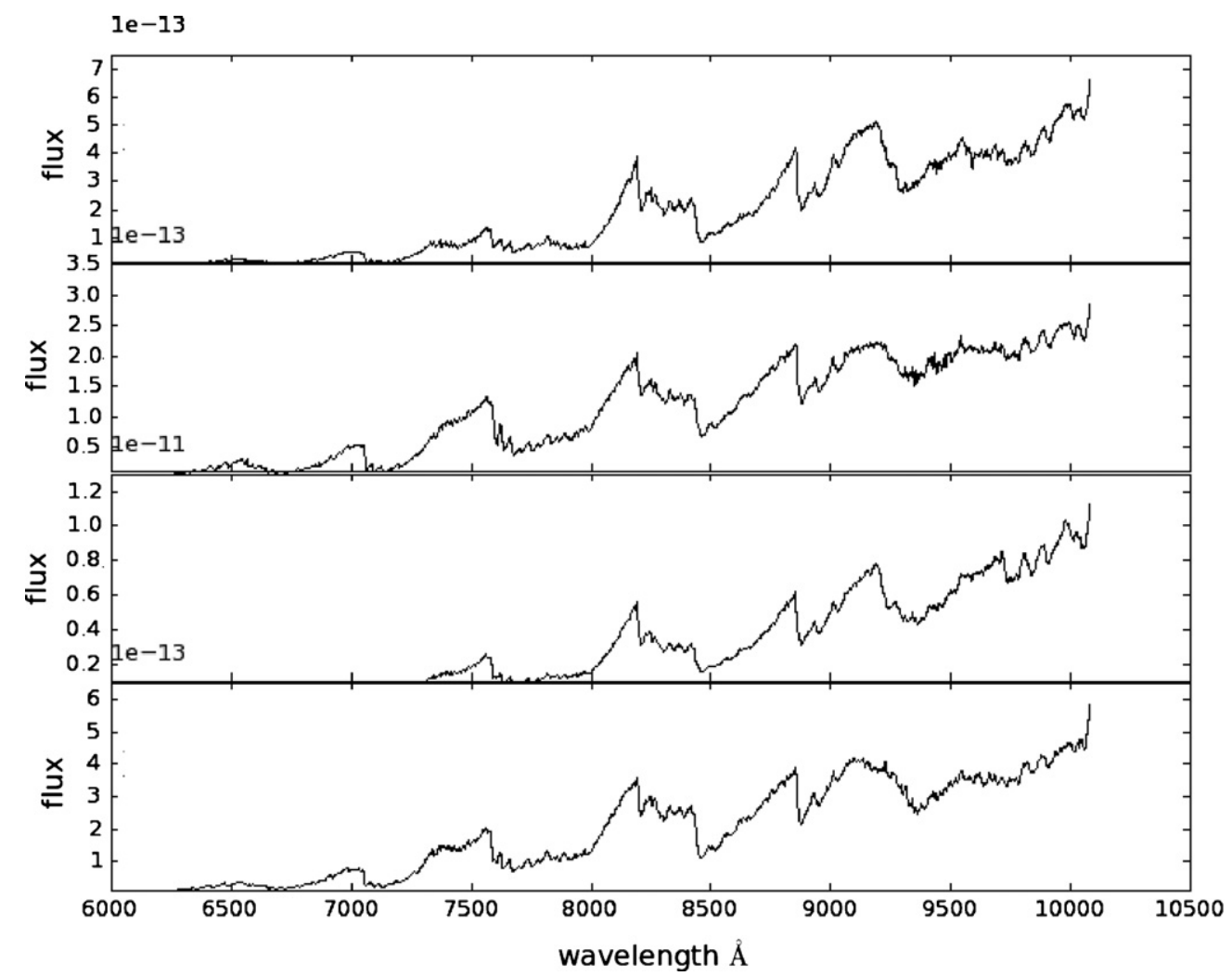

Figure 5. Four of the spectra used to compare colors to our IGS candidates. The resulting colors are shown in Figure 4.

and Local Group formation to say this definitively, so probing the IGS sample may help us to constrain either or both of these.

If every candidate were a solar-metallicity IGS giant, they would be rare tracers of a large underlying IGS population. Assuming a single burst of star formation 10 Gyr ago and a Salpeter initial mass function (IMF), these candidates represent $O\left(10^{-4}\right)$ of the total number of IGS and $O\left(10^{-3}\right)$ of the total mass in IGS, and varies only slightly for differing choices of IMF.

It is useful to compare this to theoretical predictions of stellar ejections from the Milky Way (Kollmeier et al. 2009). Stars ejected from the galaxy center through three-body interactions with an SMBH will typically have much higher metallicity than stars that were stripped from satellite galaxies originating in the outskirts of a galaxy halo (e.g., Jacoby \& Ciardullo 1999; Kirby et al. 2008). For example, if we assume that all of the IGS are solar-metallicity hypervelocity stars ejected by three-body interactions with a binary black hole consisting of an SMBH and an intermediate mass black hole (IMBH), then the total mass in stellar ejecta will be roughly equal to the mass of the IMBH (Yu \& Tremaine 2003; Quinlan 1996). Given the Milky Way SMBH mass of $4 \times 10^{6} M_{\odot}$ (Ghez et al. 2008), we would require an IMBH with mass roughly $10^{5} M_{\odot}$ as the companion, independent of initial mass function. This IMBH mass is similar to the mass of the IMBH proposed to be responsible for ejecting stars in the central region of the Galaxy (Lang et al. 2011). This yields several IGS per square degree of sky and roughly tens of red giant hypervelocity IGS in the SDSS footprint.

Similar back of the envelope calculations suggest that there are $O(1000) \mathrm{L}$ dwarfs located in the SDSS footprint, and realizing that late-type dwarfs are more common in general, we anticipate that the majority of our IGS candidates are likely L dwarfs. If these IGS candidates do turn out to be L dwarfs, then we have identified L-type dwarfs at distances of 100-200 pc, which is up to four times farther than currently known (Schmidt et al. 2010).

In an attempt to determine if the IGS sample has a distinct spatial distribution, we conducted a set of two-dimensional (2D) K-S tests that compared our candidates with template samples drawn from three characteristic distributions: (1) an exponential disk with a scale height of $300 \mathrm{pc}$ to mimic an old stellar population, and a distance cut off of 200 parsec to resemble an L dwarf distribution; (2) a random distribution; (3) and a set of observed hypervelocity stars (Brown et al. 2009). We convolved each template data set with the SDSS footprint and employed the same galactic latitude cut as in our IGS sample. Assuming that these are very cool dwarfs, the IGS sample should exhibit the same distribution on the sky as the exponential disk, but the $2 \mathrm{D} \mathrm{K}-\mathrm{S}$ test revealed otherwise: the probability that these two samples come from the same underlying distribution is only $10^{-4}$. This is ultimately not surprising since we removed any objects with a measurable proper motion, strongly selecting against L dwarfs within the disk. The second test with a random distribution resulted in an even smaller probability of $10^{-5}$, while the third test with the hypervelocity sample yielded a somewhat higher probability of $10^{-2}$. Figure 6 shows the position of our IGS candidates on the sky compared to the hypervelocity sample used here.

\section{SUMMARY AND CONCLUSIONS}

We identified 677 intragroup stellar candidates from the SDSS DR7 using color cuts based on solar-metallicity spectral standards. These are extremely red stars with $1.3<i-z<2.2$, though the color would shift bluer with lower metallicity. As shown in Figure 2, the $\mathrm{M}$ giants in the region are not completely isolated. The latest $\mathrm{M}$ dwarfs and early L dwarfs are possible sources of contamination. 


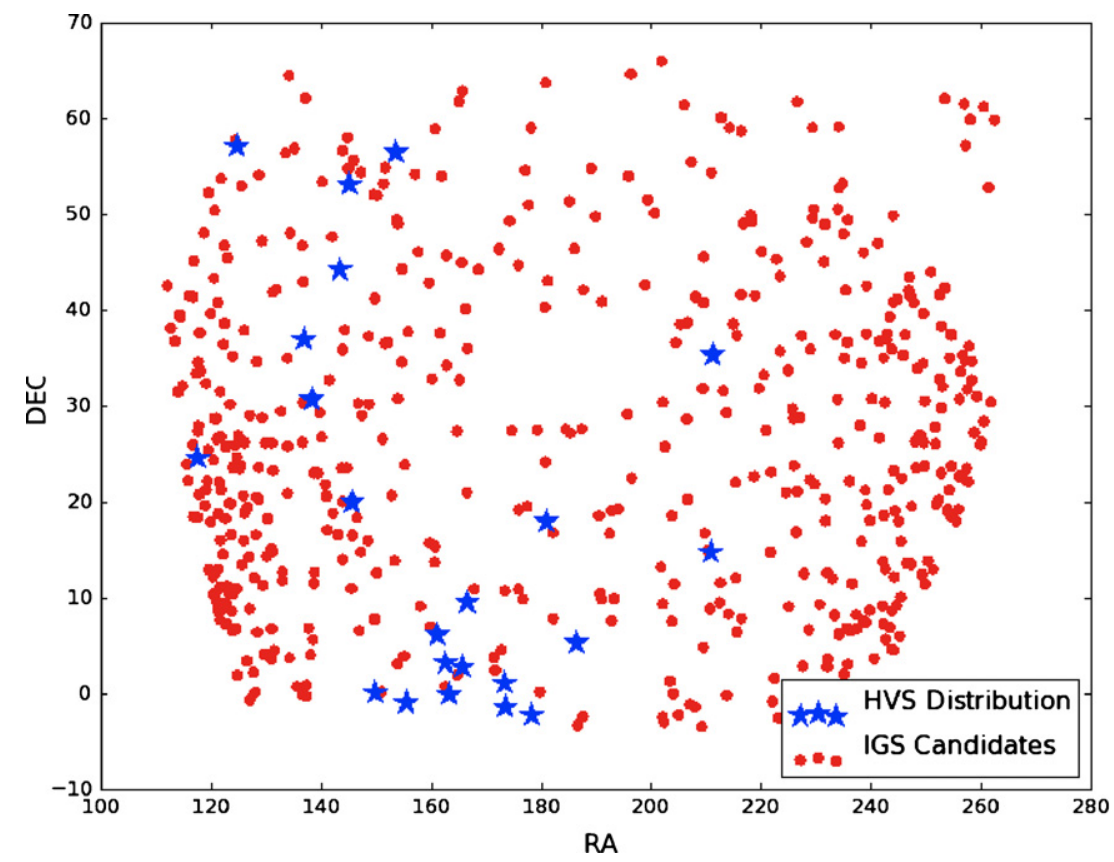

Figure 6. Relative positions of our IGS candidates and the hypervelocity stars of Brown et al. (2009) that were compared with the 2D K-S test. The comparison was made between 522 IGS candidates, red dots, and $22 \mathrm{HVS}$, blue stars. Note the higher density of IGS candidates at the edges of the footprint signifying larger numbers of these stars at lower Galactic latitudes.

Follow-up photometric observations of our candidates in the near to mid-infrared wavelengths may differentiate between late dwarfs and M giants. Future follow-up photometric observations with a $4 \mathrm{~m}$ class telescope may be promising, albeit impractical. For example, the FLAMINGOS instrument on the NOAO $4 \mathrm{~m}$ telescope could image all of our candidates with a $113 \mathrm{hr}$ total exposure time in each of the $J$ and $H$ bands, and over $600 \mathrm{hr}$ of total exposure time in the $K$ band for a $10 \sigma$ detection, while this likely would not be sufficient to distinguish $\mathrm{M}$ giants from dwarfs. Similarly, the $J, H$, and $K$ magnitude limits of NIRI on Gemini are appropriate for our targets, although would require a prohibitively long total exposure time of $2031 \mathrm{hr}$ to achieve a signal-to-noise ratio of about 12.

Also, it is possible with long-term photometric follow-up observations on a $1 \mathrm{~m}$ class telescope to differentiate between dwarfs and giants based on variability, as late-type $M$ giants tend to be highly variable.

Naturally, low resolution spectroscopic follow-up observations of these IGS candidates would be ideal to confirm their luminosity class. The Calcium II Triplet (CaT) feature at $8498 \AA$, $8542 \AA$, and $8662 \AA$ is particularly useful for distinguishing late $\mathrm{M}$ dwarfs from giants, being much more prominent in the spectra of late-type dwarfs (Reid \& Hawley 2005). In addition, the strength of the Calcium Hydride $(\mathrm{CaH})$ feature between $6800 \AA$ and $7000 \AA$ is a good indicator of luminosity class (Cohen 1978).

Once the confirmation is complete, we can test the efficiency of our color selection technique, which will be useful for large data surveys like LSST. In fact, two surveys set to launch in the coming year will be particularly well tuned to find IGS M giants. The DECam survey on the CTIO $4 \mathrm{~m}$ telescope will observe over $1000 \mathrm{deg}^{2}$, with magnitude limits of $r=23.4$, $i=24.0$, and $z=22.9$ - over two magnitudes deeper than SDSS in $z$. An even deeper survey will launch on the Subaru telescope; the HyperSuprimeCam plans to observe $2000 \mathrm{deg}^{2}$ down to $z=24.9$ and $y=23.7$. Eventually, deeper observations of IGS can reveal the metallicity of these stars - an important clue to their original birthplace within the group or in situ in the intergalactic medium.

We acknowledge Andreas Berlind for useful suggestions and for allowing us to borrow an SDSS footprint mask code. L.E.P. was supported by GAANN. K.H.B. acknowledges support from NSF Award AST-0847696. J.J.F. acknowledges support from NSF Award AST-0807873.

\section{REFERENCES}

Abadi, M. G., Navarro, J. F., \& Steinmetz, M. 2006, MNRAS, 365, 747 Aguerri, J. A. L., Gerhard, O. E., Arnaboldi, M., et al. 2005, AJ, 129, 2585 Belokurov, V., Zucker, D. B., Evans, N. W., et al. 2006, ApJ, 642, L137 Bessell, M. S., \& Brett, J. M. 1988, PASP, 100, 1134

Blanton, M. R., Brinkmann, J., Csabai, I., et al. 2003, AJ, 125, 2348 Brown, W. R., Geller, M. J., \& Kenyon, S. J. 2009, ApJ, 690, 1639 Brown, W. R., Geller, M. J., Kenyon, S. J., \& Kurtz, M. J. 2005, ApJ, 622, L33 Burgasser, A. J., Cruz, K. L., Cushing, M., et al. 2010, ApJ, 710, 1142 Byrd, G., \& Valtonen, M. 1990, ApJ, 350, 89

Chou, M., Majewski, S. R., Cunha, K., et al. 2007, ApJ, 670, 346 Cohen, J. G. 1978, ApJ, 221, 788

Conroy, C., Wechsler, R. H., \& Kravtsov, A. V. 2007, ApJ, 668, 826 Covey, K. R., Ivezić, Ž., Schlegel, D., et al. 2007, AJ, 134, 2398

Durrell, P. R., Ciardullo, R., Feldmeier, J. J., Jacoby, G. H., \& Sigurdsson, S. 2002, ApJ, 570, 119

Fan, X., Strauss, M. A., Richards, G. T., et al. 2001, AJ, 121, 31

Feldmeier, J. J., Ciardullo, R., Jacoby, G. H., \& Durrell, P. R. 2004a, ApJ, 615, 196

Feldmeier, J. J., Mihos, J. C., Morrison, H. L., et al. 2004b, ApJ, 609, 617

Feldmeier, J. J., Mihos, J. C., Morrison, H. L., Rodney, S. A., \& Harding, P. 2002, ApJ, 575, 779

Fukugita, M., Ichikawa, T., Gunn, J. E., et al. 1996, AJ, 111, 1748

Ghez, A. M., Salim, S., Weinberg, N. N., et al. 2008, ApJ, 689, 1044

Gunn, J. E., Carr, M., Rockosi, C., et al. 1998, AJ, 116, 3040

Gunn, J. E., Siegmund, W. A., Mannery, E. J., et al. 2006, AJ, 131, 2332

Hawley, S. L., Covey, K. R., Knapp, G. R., et al. 2002, AJ, 123, 3409

Hills, J. G. 1988, Nature, 331, 687

Hogg, D. W., Finkbeiner, D. P., Schlegel, D. J., \& Gunn, J. E. 2001, AJ, 122, 2129

Holley-Bockelmann, K., Sigurdsson, S., Mihos, J. C., et al. 2005, arXiv: astro-ph/0512344

Ibata, R., Lewis, G. F., Irwin, M., Totten, E., \& Quinn, T. 2001, ApJ, 551, 294 
Ibata, R., Martin, N. F., Irwin, M., et al. 2007, ApJ, 671, 1591

Ivezić, Ž., Smith, J. A., Miknaitis, G., et al. 2007, AJ, 134, 973

Jacoby, G. H., \& Ciardullo, R. 1999, ApJ, 515, 169

Kirby, E. N., Simon, J. D., Geha, M., Guhathakurta, P., \& Frebel, A. 2008, ApJ, $685, \mathrm{~L} 43$

Kollmeier, J. A., Gould, A., Knapp, G., \& Beers, T. C. 2009, ApJ, 697, 1543

Krick, J. E., \& Bernstein, R. A. 2007, AJ, 134, 466

Laidler, V., et al. 2005, Synphot User's Guide, Version 5.0 (Baltimore, MD: STScI)

Lang, M., Holley-Bockelmann, K., Bogdanovic, T., Amaro-Seoane, P., \& Sesana, A. 2011, arXiv:1107.2923

Lawrence, A., Warren, S. J., Almaini, O., et al. 2007, MNRAS, 379, 1599

Lee, M. G., Park, H. S., \& Hwang, H. S. 2010, Science, 328, 334

Magorrian, J., \& Tremaine, S. 1999, MNRAS, 309, 447

Majewski, S. R., Skrutskie, M. F., Weinberg, M. D., \& Ostheimer, J. C. 2003, ApJ, 599, 1082

Mateo, M., Olszewski, E. W., \& Walker, M. G. 2008, ApJ, 675, 201

McConnachie, A. W., Irwin, M. J., Ibata, R. A., et al. 2009, Nature, 461, 66

Merritt, D. 1984, ApJ, 276, 26

Mihos, C. 2003, arXiv:astro-ph/0305512

Mihos, J. C. 2004, in IAU Symp. 217, Recycling Intergalactic and Interstellar Matter, ed. P. A. Duc, J. Braine, \& E. Brinks (San Francisco, CA: ASP), 390

Mihos, J. C., Harding, P., Feldmeier, J., \& Morrison, H. 2005, ApJ, 631, L41

Moore, B., Katz, N., Lake, G., Dressler, A., \& Oemler, A. 1996, Nature, 379, 613

Murante, G., Arnaboldi, M., Gerhard, O., et al. 2004, ApJ, 607, L83
Murante, G., Giovalli, M., Gerhard, O., et al. 2007, MNRAS, 377, 2

Napolitano, N. R., Pannella, M., Arnaboldi, M., et al. 2003, ApJ, 594, 172

Oke, J. B., Cohen, J. G., Carr, M., et al. 1995, PASP, 107, 375

Peng, E. W., Ferguson, H. C., Goudfrooij, P., et al. 2011, ApJ, 730, 23

Pickles, A. J. 1998, PASP, 110, 863

Quinlan, G. D. 1996, New Astron., 1, 35

Reid, I. N., \& Hawley, S. L. 2005, in New Light on Dark Stars: Red Dwarfs, Low-mass Stars, Brown Dwarfs, ed. I. N. Reid \& S. L. Hawley (Chichester, UK: Praxis)

Richards, G. T., Fan, X., Newberg, H. J., et al. 2002, AJ, 123, 2945

Rudick, C. S., Mihos, J. C., Frey, L. H., \& McBride, C. K. 2009, ApJ, 699, 1518

Sales, L. V., Navarro, J. F., Abadi, M. G., \& Steinmetz, M. 2007, MNRAS, 379, 1475

Schlegel, D. J., Finkbeiner, D. P., \& Davis, M. 1998, ApJ, 500, 525

Schmidt, S. J., West, A. A., Hawley, S. L., \& Pineda, J. S. 2010, AJ, 139, 1808

Sesana, A., Haardt, F., \& Madau, P. 2006, ApJ, 651, 392

Skiff, B. A. 2010, VizieR Online Data Catalog, 1, 2023

Smith, M. C., Ruchti, G. R., Helmi, A., et al. 2007, MNRAS, 379, 755

Williams, B. F., Ciardullo, R., Durrell, P. R., et al. 2007, ApJ, 656, 756

Worthey, G., \& Lee, H.-c. 2011, ApJS, 193, 1

Yanny, B., Newberg, H. J., Kent, S., et al. 2000, ApJ, 540, 825

York, D. G., Adelman, J., Anderson, J. E., Jr., et al. 2000, AJ, 120, 1579

Yu, Q., \& Tremaine, S. 2003, ApJ, 599, 1129

Zucker, D. B., Kniazev, A. Y., Martínez-Delgado, D., et al. 2007, ApJ, 659, L21 